\title{
A CO-OPERATIVE METHODOLOGY TO ESTIMATE CAR FUEL CONSUMPTION BY USING SMARTPHONE SENSORS
}

\author{
Vittorio Astarita, Giuseppe Guido, Domenico Mongelli, Vincenzo Pasquale Giofrè \\ Dept of Civil Engineering, University of Calabria, Italy
}

Submitted 29 November 2014; resubmitted 26 February 2015; accepted 17 June 2015

\begin{abstract}
The European Commission has recently promoted research programs aimed at finding solutions to the ever more compelling problem of air pollution from road vehicles and has also indicated a better sustainability among the possible impacts of co-operative Intelligent Transportation Systems. In fact, many practical solutions can be developed that allow drivers and management to optimise resources and to contain costs and the emissions of pollutants by applying communication systems between vehicles (Vehicle-to-Vehicle - V2V) and between vehicles and infrastructure (Vehicle-to-Infrastructure - V2I). Along this mainstream this paper present a co-operative system which offer drivers the ability to manage their consumption and driving style, suggesting corrections to the usually adopted behaviour. The new contribution of this paper is both the co-operative approach between drivers to achieve a common goal of a better common energy consumption strategy and a methodology to estimate fuel consumption just by using Satellite data obtained from a simple smartphone. Since the fuel consumption has to be evaluated with regards to the specific vehicle type the system is based also on crowdsourcing of the specific vehicle consumption performances. The paper describes a system that gathers data on fuel consumption from the co-operating drivers that can build together the data set necessary to the system itself once they accept this paper paradigm: crowd sourced co-operation for a smarter and more sustainable transport system.
\end{abstract}

Keywords: fuel consumption; traffic flow; smartphone; sustainable transportation; co-operative systems.

\section{Introduction}

Energy consumption, especially regarding the transport component, is receiving more and more attention worldwide. Researchers are addressing their efforts to minimize the environmental impact generated by road traffic congestion. Several research projects were recently promoted by European Commission to find solutions useful to users and car manufacturers (Smart, Green and Integrated Transport societal challenge from Horizon 2020 Program). This paper introduces a procedure for the evaluation of fuel consumption of road vehicles on the basis of kinematic parameters (speed and acceleration) detected with embedded GPS smartphone technology. The procedure aims to collect crowd-sourced data by using co-operating drivers. The idea is based on the Web 2.0 concept for exchanging and sharing information through a shared platform. The system has a wikilike environment for exchanging and sharing information among users, and can perform information collection, data fusion and dissemination through a geo-localization of drivers mobile smartphones. In the proposed system a co-operative approach is used as in Lee et al.
(2010) and Figueiredo et al. (2001). The paper is organized as follows. The Section 1 provides a state of the art of the systems actually used to automatically evaluate the energy consumption of the vehicles. The Section 2 is focused on the methodology used to analyse the vehicles' fuel consumption based on speed, acceleration and slope profiles. Section 3 introduces an experimentation test of the proposed methodology. Section 4 describes a statistical analysis of the experimental results. Section 5 describes the co-operative system. The paper concludes with a discussion on the preliminary results and on future developments of the research.

\section{Literature Review}

Fuel consumption models are currently developed and calibrated; however, some of them do not evaluate fuel consumption through data coming from the vehicle control unit, but indirectly based on the amount of fuel available, the mileage and the tyre pressure to produce an overall general estimate of fuel consumption that is not instantaneous. These systems are not optimized to help drivers in reducing their fuel consumption; some of

Corresponding author: Vittorio Astarita

E-mail: vittorio.astarita@unical.it 
these are purely diagnostic in nature, while others produce only qualitative indicators.

Some researchers proposed a C-ITS applications to improve traffic control and management (Martinez et al. 2010; An et al. 2011). A great effort has been made to improve the co-operative systems by using mobile radio networks (Andrisano et al. 2000), wireless communications technologies (Kosch et al. 2009), and integration among traffic, communication and multi-user driving simulator framework (Katsaros et al. 2011). Other researchers are working on a new systems by using mobile technologies to improve the quality of information to be shared among the road users. In Tulusan et al. (2012) eco-feedback technologies providing information on the driving behavior have shown to be an effective means to suggest changes in driving style and reduce costs and environmental impacts. Araujo et al. (2012) presented a new methodology for influencing the drivers' behaviour based on smartphone application that helps drivers to reduce fuel consumption. In this research, smartphone's sensors are used to detect the vehicle state and the driving pattern suggesting new behaviours in real time that will lead to a more efficient driving experience. Corti (2013) suggests to compute the vehicle's energy consumption using a parametric analytical model, which depends on speed and acceleration gathered via inertial measurements through the embedded inertial sensors of a smartphone. Oehlerking (2011) explores several applications of mobile and vehicle sensors to investigate the evaluation of fuel consumption. He developed a framework for a crowdsourcing platform that can process OBD2 (On-Board Diagnostic) and smartphone sensor data to use them in a traffic-themed application layer.

\section{Methodology of Experimental Investigations}

The methodology developed to evaluate fuel consumption (Astarita et al. 2014a, 2014b, 2014c) is based on the principle that the rate of engine power that defines fuel consumption, on a specific infrastructure's segment, in specific traffic conditions and with specific characteristics of driving style, depends mainly on speed, acceleration values and road slope. Hence the presented methodology acquires the motion characteristics of the vehicle (speed-time data from satellite GPS and GLONASS system), and evaluates the fuel consumption on the basis of the measured speeds and accelerations. The idea is to have a preliminary survey phase, based on the simultaneous acquisition of instantaneous engine's data and kinematics parameters (to be conducted for each vehicle or class of vehicles), that would fill up a multidimensional consumption matrix containing the real average values of fuel consumption for each triple value of speed-acceleration-slope (Fig. 1).

The methodology to evaluate fuel consumption is based on the collection of two different types of data: (1) those coming from the vehicle engine, through a communication OBD2 interface, and (2) those coming from the satellite systems, through the use of mobile devices with positioning sensors. During the survey phase, the position and the speed of the vehicle are read through satellite systems; simultaneously, by using the OBD2 Bluetooth interface, the information on real instantaneous fuel consumption is also acquired from the vehicle engine. This has been done by using discretized intervals of speed and acceleration and three different slope categories (uphill, downhill and flat). In other words, in the survey phase, based on all the measured consumptions, for every measured speed-acceleration couple (and slope category) an average of fuel consumptions is calculated. The average fuel consumption associated with every speed-acceleration couple (and slope category) is based on values that are measured every second.

Once the consumption matrix is obtained, the methodology applies the matrix to make estimations based just on smartphone GPS data (speed, acceleration and slope). The smartphone positioning data become the base to evaluate (for every second) the effective triple value of speed-acceleration-slope at which the car is moving. The corresponding values of the consumption matrix are then used to estimate the instantaneous consumption when OBD data are not available.

The methodology can be implemented in different ways taking into account different discretization intervals for speed, acceleration and slope. The smaller the discretization intervals the more the procedure would be reliable by reducing discretization errors. Of course,

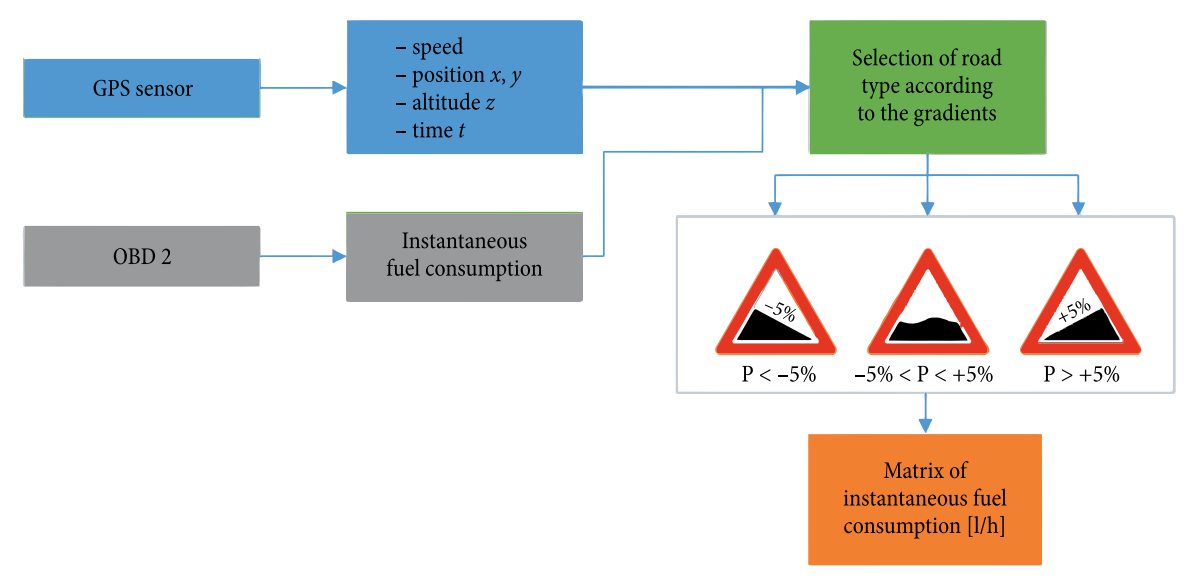

Fig. 1. Methodology for defining the matrix of fuel consumption 
even with a perfect discretization many other variables that contribute to fuel consumption still would be present and would affect the accuracy of the estimation results. The presented methodology is not able to give a perfect estimation of fuel consumption yet it can be useful for many practical applications with the aim of moving drivers toward more sustainable driving styles. A more detailed discretization would require a more complicated survey phase, so there is a trade-off between ease of applicability of the procedure and the accuracy in the estimations. In this paper, three case studies are presented with a quite large discretization. However, these case studies showed quite good results in the accuracy of the estimations.

In the survey phase the matrix values are constantly updated bringing up more reliable values when more measures are performed. In the test application the speed has been decomposed into 15 classes, from 0 to over $135 \mathrm{~km} / \mathrm{h}$, while acceleration in 17 classes from values lower than $-15 \mathrm{~m} / \mathrm{s}^{2}$ to values higher than $+15 \mathrm{~m} / \mathrm{s}^{2}$.

The fuel consumption matrix will be used only to give the value of the instantaneous fuel consumption of the vehicle without the need of any traditional connections like OBD2 devices or else with the engine data. At any time instant $(t)$, the value of the estimated Instantaneous Fuel Consumption (IFC) will be obtained as a function of speed and acceleration:

$$
\operatorname{IFC}(t)=f(\operatorname{speed}(t), \operatorname{acc}(t)) .
$$

The Total Fuel Consumption (TFC) is calculated as:

$$
T F C=\sum_{i=1}^{n}(I F C)_{i} \cdot \Delta t_{i}
$$

where: $\Delta t_{i}$ is the time increment value (1 second) in which the instantaneous fuel consumption is assumed constant; $n$ is the number of time increment values included in the travel time.

\section{Results of Experiments}

The experimentation was conducted on different vehicles with Diesel and GPL fuel. The experiment results presented in this paper are related to a Peugeot 307 HDI with a $2000 \mathrm{cc}$ diesel engine (Diesel 1), a Nissan Pixo with a $1000 \mathrm{cc}$ GPL engine (GPL) and a Fiat Doblo with a 2000cc diesel Multijet engine (Diesel 2). Each of the vehicles run in the survey phase more than 100 kilometres, so as to evaluate the reliability of the model on a medium distance. Thirty-six trips were made (about $325 \mathrm{~km}$ ) with the Diesel 1 vehicle, thirty-three trips were made (about $387 \mathrm{~km}$ ) with the GPL vehicle and nineteen trips were made (about $128 \mathrm{~km}$ ) with Diesel 2 vehicle.

It must be emphasised that the consumption matrix has not been updated constantly while driving, but only at the end of the trip. Furthermore, for each vehicle, a first trip of at least $25 \mathrm{~km}$ was performed, used for a preliminary filling of the fuel consumption matrix.

\begin{tabular}{|c|c|c|c|c|c|c|c|c|c|c|c|c|}
\hline \multirow[b]{2}{*}{$\begin{array}{l}\text { Trip } \\
\text { No }\end{array}$} & \multicolumn{4}{|c|}{ Diesel 1} & \multicolumn{4}{|c|}{ Diesel 2} & \multicolumn{4}{|c|}{ GPL } \\
\hline & $\begin{array}{c}\text { Real }^{1} \\
{[\mathrm{~L}]}\end{array}$ & $\begin{array}{c}\text { Estimated }^{2} \\
{[\mathrm{~L}]}\end{array}$ & $\begin{array}{c}\text { Error }^{3} \\
{[\%]}\end{array}$ & $\begin{array}{c}\text { Distance }^{4} \\
{[\mathrm{~km}]}\end{array}$ & $\begin{array}{c}\text { Real }^{1} \\
{[\mathrm{~L}]}\end{array}$ & $\begin{array}{c}\text { Estimated }^{2} \\
{[\mathrm{~L}]}\end{array}$ & $\begin{array}{c}\text { Error }^{3} \\
{[\%]}\end{array}$ & $\begin{array}{c}\text { Distance }^{4} \\
{[\mathrm{~km}]}\end{array}$ & $\begin{array}{c}\mathrm{Real}^{1} \\
{[\mathrm{~L}]}\end{array}$ & $\begin{array}{c}\text { Estimated }^{2} \\
{[\mathrm{~L}]}\end{array}$ & $\begin{array}{c}\text { Error }^{3} \\
{[\%]}\end{array}$ & $\begin{array}{c}\text { Distance }^{4} \\
{[\mathrm{~km}]}\end{array}$ \\
\hline 1 & 1.89 & 2.22 & -17.1 & 15.6 & 0.31 & 0.27 & 12.5 & 1.5 & 0.42 & 0.51 & -22.6 & 5.6 \\
\hline 2 & 1.60 & 1.70 & -5.9 & 13.1 & 0.57 & 0.58 & -2.1 & 3.6 & 3.38 & 3.47 & -2.6 & 60.9 \\
\hline 3 & 1.81 & 1.75 & 3.1 & 13.6 & 0.24 & 0.23 & 2.1 & 1.6 & 2.44 & 2.39 & 2.0 & 42.6 \\
\hline 4 & 1.64 & 1.37 & 16.5 & 10.8 & 0.72 & 0.76 & -5.6 & 4.3 & 0.54 & 0.61 & -12.7 & 6.6 \\
\hline 5 & 1.58 & 1.45 & 8.5 & 10.9 & 0.24 & 0.26 & -9.8 & 1.5 & 0.74 & 0.77 & -4.2 & 8.7 \\
\hline 6 & 1.55 & 1.41 & 9.3 & 10.7 & 0.84 & 0.91 & -7.2 & 5.9 & 0.79 & 0.69 & 12.2 & 6.0 \\
\hline 7 & 1.77 & 1.31 & 25.7 & 10.3 & 0.78 & 0.73 & 6.9 & 4.3 & 0.41 & 0.36 & 11.2 & 4.7 \\
\hline 8 & 0.98 & 1.15 & -17.3 & 9.0 & 0.47 & 0.55 & -17.8 & 3.4 & 0.87 & 0.73 & 15.7 & 10.5 \\
\hline 9 & 0.81 & 0.95 & -16.9 & 7.0 & 0.25 & 0.26 & -1.8 & 1.6 & 0.52 & 0.52 & 0.8 & 5.8 \\
\hline 10 & 1.34 & 1.07 & 20.1 & 8.5 & 0.68 & 0.68 & 0.0 & 4.2 & 0.41 & 0.46 & -13.6 & 5.8 \\
\hline 11 & 1.11 & 1.05 & 6.2 & 8.9 & 0.68 & 0.74 & -8.6 & 4.8 & 0.48 & 0.54 & -12.8 & 5.6 \\
\hline 12 & 1.02 & 1.03 & -0.5 & 8.6 & 0.96 & 0.82 & 14.1 & 4.4 & 0.75 & 0.65 & 14.5 & 8.6 \\
\hline 13 & 0.47 & 0.51 & -6.8 & 2.9 & 0.77 & 0.73 & 5.1 & 4.7 & 1.22 & 1.01 & 17.2 & 12.0 \\
\hline 14 & 0.57 & 0.51 & 10.9 & 3.3 & 6.14 & 5.81 & 5.4 & 40.0 & 0.36 & 0.37 & -2.9 & 3.9 \\
\hline 15 & 1.41 & 1.13 & 19.3 & 8.3 & 3.15 & 3.56 & -13.2 & 27.0 & 0.26 & 0.28 & -5.1 & 3.6 \\
\hline 16 & 1.48 & 1.38 & 7.1 & 9.6 & 0.83 & 0.84 & -1.6 & 5.3 & 0.23 & 0.28 & -20.2 & 1.9 \\
\hline 17 & 0.22 & 0.26 & -16.5 & 1.6 & 0.83 & 0.83 & -0.5 & 5.6 & 0.75 & 0.89 & -19.3 & 10.0 \\
\hline 18 & 1.72 & 1.87 & -8.7 & 14.8 & 0.53 & 0.49 & 6.6 & 2.4 & 0.62 & 0.49 & 21.1 & 6.2 \\
\hline 19 & 1.74 & 1.75 & -1.0 & 13.4 & 0.41 & 0.33 & 19.0 & 2.1 & 0.94 & 0.80 & 14.7 & 9.8 \\
\hline
\end{tabular}

Table. Results obtained from the experiments

Notes: ${ }^{1}$ real total fuel consumption by trip; ${ }^{2}$ estimated total fuel consumption by trip; ${ }^{3}$ percentage error between real and estimated total fuel consumption; ${ }^{4}$ length of the trip. 
All trips have been made trying to capture the largest number of possible road type cases and different possible acceleration-speed couples. The Table shows some results obtained from the trips completed by the three vehicles.

\section{Statistical Analysis of Results}

From the analysis of the real fuel consumption for each trip and the estimated one, it is possible to see that estimates are quite good considering the simplicity of the system and the length of the trips that were performed in urban areas with a lot of variability in driving conditions.

The effectiveness of the methodology has been assessed by the standard deviation of the percentage errors Eq. (3) in the estimation of total fuel consumption. Estimates have been obtained from the fuel consumption matrix updated to the last trip made before the current trip:

$$
S D=\sqrt{\frac{\sum_{i=1}^{n}(\text { error }-\overline{\text { error }})^{2}}{n-1} .} .
$$

From this analysis (Fig. 2) it can be seen that, after the initials trips, the standard deviation trend assumes a more regular decreasing configuration, with values that stabilize on $13.5 \%$ for Diesel 1, 12.5\% for GPL and 9.5\% for Diesel 2.

By way of example, in the Fig. 3 the fuel consumption matrix is illustrated for the Diesel 2 vehicle.

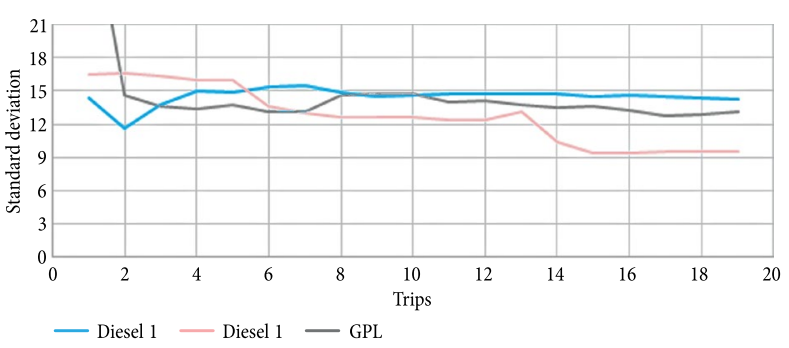

Fig. 2. Standard deviation trend of the percentage error

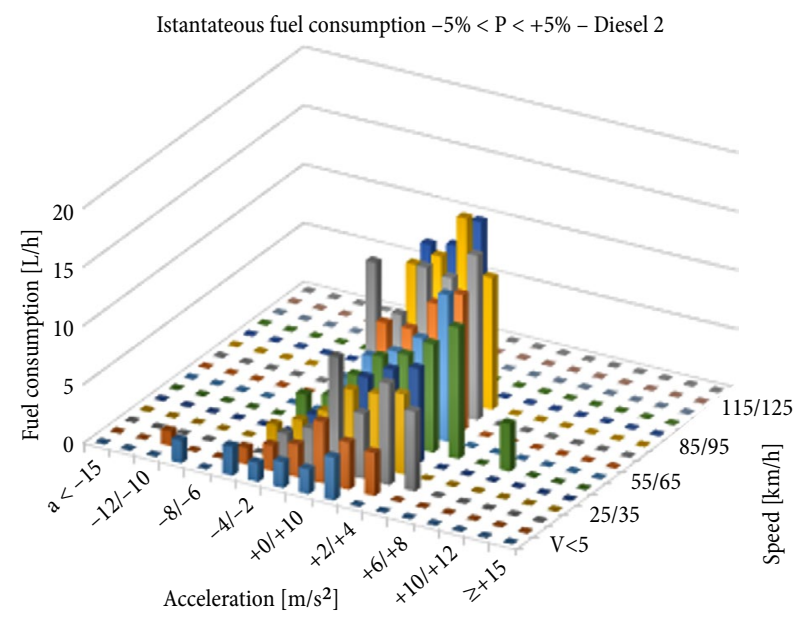

Fig. 3. Fuel consumption matrix for Diesel 2 vehicle

\section{Toward a Co-Operative System}

This methodology has been implemented in a client/ server platform. The system bases its operation by coupling smartphones and a GIS Web server system where the mobile device is used to record, store data and send them to the server, while the GIS Web server, operating on a Linux-MySQL-PHP platform, processes them, and returns statistics in graphical form. Fuel consumption matrices are filled with the concept of self-learning. In fact, drivers can decide to load data in the system by connecting the smartphone through a Bluetooth OBD2 communication device to the engine control unit obtaining consumption values for the specific vehicle. The self-learning mechanism can take place if the OBD2 device is connected to the vehicle during driving. The mobile Apps aims to store this data and upload them to the system so to co-operate and build the matrix of consumption for a specific vehicle (or for a set of similar vehicles). At the end of the trip, the mobile Apps shows the travel statistics on several information, such as average speed, maximum speed, travel time, and the total fuel consumption.

The system therefore can allow users to keep under control fuel consumption and to improve driving trip after trip toward a more sustainable driving style. The driver also has the option to visualize, on the map, aggregate information, querying the web server on a group of trips, rather than just on a single trip. This kind of elaborated visualization allow users to co-operate and understand how they can improve their driving style.

\section{Conclusions}

The proposed system is based on a new methodology for evaluating fuel consumption on the basis of vehicles kinematics detected with smartphone technology. The higher the level of penetration of the system the greater the level of data in the matrices, and therefore the accuracy of the procedure. A dedicated Apps for smartphone helps users in choosing eco and safe driving behaviors and suggests strategies to be shared with the community. The proposed system allows drivers to analyze detailed information about their individual driving style and statistics on their fuel consumption. This study underscores the usefulness of the smartphone technology for improving Co-operative-ITS and providing solutions to reduce energy consumption. The preliminary results of this study show that the proposed system provides sufficient reliable estimates of fuel consumption and a potential great support to the users' choices with respect to driving styles to be adopted. Particularly, the standard deviation trend of the percentage errors in the estimation of total fuel consumption assumes a more regular decreasing configuration, with values that stabilize on 13.5\% for Diesel 1, 12.5\% for GPL and 9.5\% for Diesel 2. The co-operating drivers relying on the system can build together the data set necessary to the system itself once they accept this paper paradigm: crowd sourced information and co-operation for a smarter and more sustainable transport system. 


\section{References}

An, S.-H.; Lee, B.-H.; Shin, D.-R. 2011. A survey of intelligent transportation systems, in 2011 Third International Conference on Computational Intelligence, Communication Systems and Networks (CICSyN), 26-28 July 2011, Bali, Indonesia, 332-337. http://dx.doi.org/10.1109/CICSyN.2011.76

Andrisano, O.; Verdone, R.; Nakagawa, M. 2000. Intelligent transportation systems: the role of third generation mobile radio networks, IEEE Communications Magazine 38(9): 144-151. http://dx.doi.org/10.1109/35.868154

Araujo, R.; Igreja, A.; De Castro, R.; Araujo, R. E. 2012. Driving coach: a smartphone application to evaluate driving efficient patterns, in 2012 IEEE Intelligent Vehicles Symposium (IV), IEEE, 3-7 June 2012, Alcalá de Henares, 1005-1010. http://dx.doi.org/10.1109/IVS.2012.6232304

Astarita, V.; Guido, G.; Mongelli, D. W. E.; Giofre V. P. 2014a. EcoSmart and TutorDrive: tools for fuel consumption reduction, 2014 IEEE International Conference on Service Operations and Logistics, and Informatics (SOLI), 8-10 October 2014, Qingdao, China, 183-187. http://dx.doi.org/10.1109/SOLI.2014.6960716

Astarita, V.; Festa, D. C.; Mongelli, D. W. E. 2014b. EcoSmart: an application for smartphones for monitoring driving economy, Advanced Materials Research 827: 360-367. http://dx.doi.org/10.4028/www.scientific.net/AMR.827.360

Astarita, V.; Festa, D. C.; Mongelli, D. W. E.; Mongelli, N.; Ruffolo, O.; Servino, A. 2014c. EcoSmart: a survey and sector analysis of mobile phone application market for fuel consumption reduction, Applied Mechanics and Materials 519-520: 1546-1555.

http://dx.doi.org/10.4028/www.scientific.net/AMM.519520.1546

Corti, A. 2013. Vehicle's Energy Estimation and Optimization via Inertial Measurements: PhD Thesis. Polytechnic University of Milan, Italy. Availabe from Internet: https://www. politesi.polimi.it/handle/10589/74254

Figueiredo, L.; Jesus, I.; Machado, J. A. T.; Ferreira, J. R.; De Carvalho, J. L. M. 2001. Towards the development of intelligent transportation systems, in 2001 IEEE Intelligent Transportation Systems: Proceedings, 25-29 August 2001, Oakland, CA, 1206-1211. http://dx.doi.org/10.1109/ITSC.2001.948835

Katsaros, K.; Kernchen, R.; Dianati, M.; Rieck, D. 2011. Performance study of a green light optimized speed advisory (GLOSA) application using an integrated cooperative ITS simulation platform, in 2011 th International Wireless Communications and Mobile Computing Conference (IWCMC), , 4-8 July 2011, Istanbul, Turkey, 918-923. http://dx.doi.org/10.1109/IWCMC.2011.5982524

Kosch, T.; Kulp, I.; Bechler, M.; Strassberger, M.; Weyl, B.; Lasowski, R. 2009. Communication architecture for cooperative systems in Europe, IEEE Communications Magazine 47(5): 116-125.

http://dx.doi.org/10.1109/MCOM.2009.4939287

Lee, W.-H.; Tseng, S.-S.; Shieh, W.-Y. 2010. Collaborative realtime traffic information generation and sharing framework for the intelligent transportation system, Information Sciences 180(1): 62-70. http://dx.doi.org/10.1016/j.ins.2009.09.004

Martinez, F. J.; Toh, C.-K.; Cano, J.-C.; Calafate, C. T.; Manzoni, P. 2010. Emergency services in future intelligent transportation systems based on vehicular communication networks, IEEE Intelligent Transportation Systems Magazine 2(2): 6-20. http://dx.doi.org/10.1109/MITS.2010.938166
Oehlerking, A. L. 2011. StreetSmart: Modeling Vehicle Fuel Consumption with Mobile Phone Sensor Data through a Participatory Sensing Framework: MSc Thesis. Massachusetts Institute of Technology, USA. 109 p. Available from Internet: http://dspace.mit.edu/handle/1721.1/68950

Tulusan, J.; Staake, T.; Fleisch, E. 2012. Providing eco-driving feedback to corporate car drivers: what impact does a smartphone application have on their fuel efficiency?, in UbiComp'12: Proceedings of the 2012 ACM Conference on Ubiquitous Computing, 5-8 September 2012, Pittsburgh, PA, 212-215. http://dx.doi.org/10.1145/2370216.2370250 